Urologe 2018· 57:1357-1359

https://doi.org/10.1007/s00120-018-0665-8

Online publiziert: 23. Mai 2018

(c) Der/die Autor(en) 2018

CrossMark

\author{
H. Griessner ${ }^{1}$ T. Kunit ${ }^{1}$ D. Colleselli ${ }^{1}$ M. Drerup ${ }^{1} \cdot$ L. Oberhammer ${ }^{1}$. \\ T. Wolfgruber ${ }^{2} \cdot$ L. Lusuardi ${ }^{\prime} \cdot$ M. Mitterberger ${ }^{1}$ \\ ' Department of Urology and Andrology, Paracelsus Medical University Salzburg, Salzburg, Austria \\ ${ }^{2}$ Department of Radiology, Paracelsus Medical University, Salzburg, Austria
}

\section{Pseudoaneurysma der bulbären Harnröhre nach traumatischer Katheterisierung}

\section{Einleitung}

Blasenkatheter werden im klinischen Alltag aus den verschiedensten Gründen häufig gelegt. Der Großteil der Anlagen erfolgt dabei völlig komplikationslos, jedoch kann v. a. bei Harnröhrenengen oder aufgrund einer Schleimhautfalte sehr leicht eine Verletzung der Harnröhre entstehen. Die traumatische Anlage eines Dauerkatheters kann zu Harnröhrenstrikturen, Fistelbildung oder in seltenen Fällen auch zur Ausbildung eines Pseudoaneurysmas führen. Während über die Bildung von Pseudoaneurysmen nach perkutaner Nephrolithotripsie eine Vielzahl an Kasuistiken existieren [2], ist die Entstehung eines Pseudoaneurysmas nach Anlage eines Blasenkatheters eine Rarität.

\section{Falldarstellung}

\section{Anamnese}

Bei einem 57-jährigen Patienten wurde eine laparoskopische Fundoplikation bei gastroösophagealen Reflux durchgeführt. Es bestanden keine wesentlichen Begleiterkrankungen. Aus urologischer Sicht hatte der Patient zu diesem Zeitpunkt keine Probleme. Intraoperativ wurde zur besseren Überwachung der Harnausscheidung ein Blasenkatheter (Nelaton Charrière 14) gelegt. Bei deutlich erschwerter Anlage des Dauerkatheters gelang es nach mehreren Versuchen, den transurethralen Katheter einzulegen. Initial zeigte sich ein deutlich blutiger Urin, welcher jedoch im weiteren Verlauf ohne Intervention wieder aufklarte. Auch im weiteren postoperativen Verlauf kam es vorerst zu keiner weiteren Blutung.

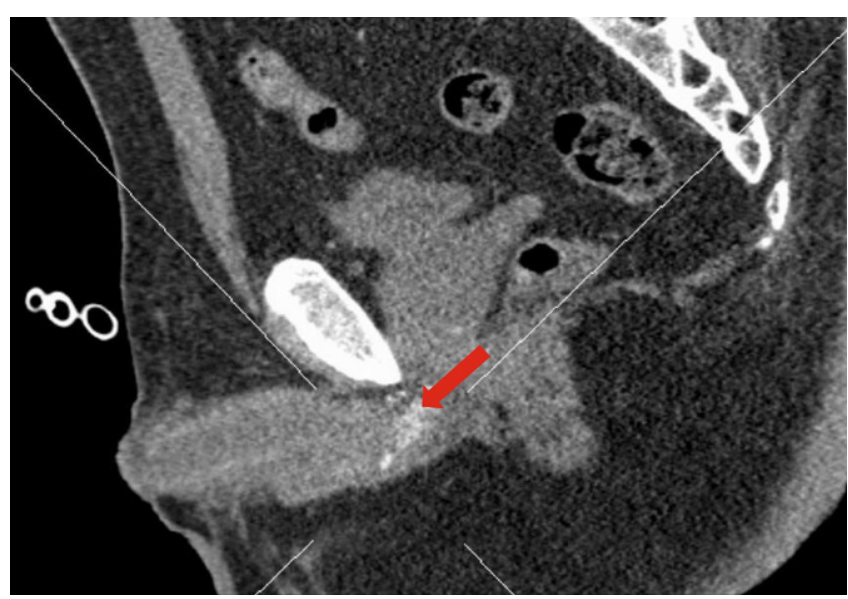

Abb. $1<$ Blutungsquelle in der Harnröhre (Pfeil) in der Kontrastmittel(KM)-Computertomographie(CT), arterielle Phase

\section{Klinischer Befund}

Zwei Wochen nach Entlassung des Patienten trat erneut eine Makrohämaturie mit konsekutiver Blasentamponade auf. Eine stationäre Aufnahme an einer urologischen Abteilung mit Ausräumung der Blasentamponade und anschließender Anlage eines Spülkatheters war notwendig. Nach passagerer Versorgung mit einem transurethralen Katheter kam es erneut zum Sistieren der Makrohämaturie und der Patient wurde abermals ohne Katheter nach Hause entlassen. Eine Woche nach Entlassung kam es wiederum zu Blutungen aus der Harn-

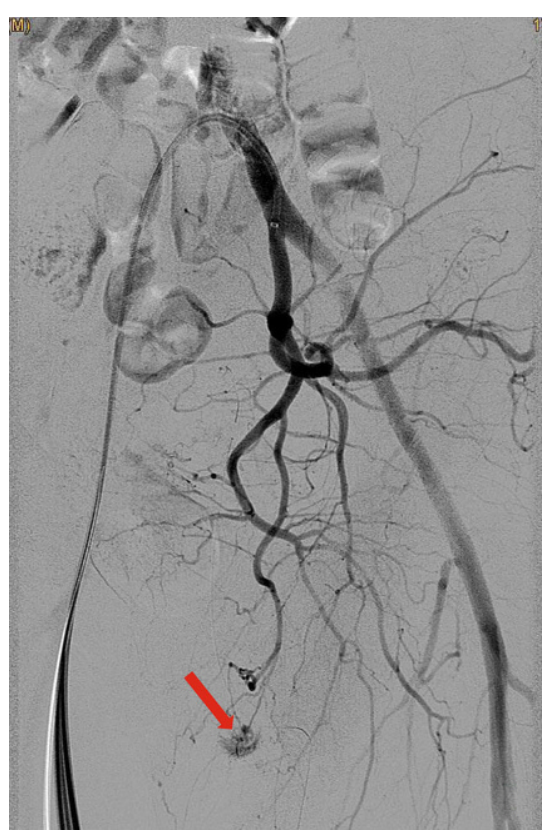

Abb. $2 \triangle$ Pseudoaneurysma der A. pudenda interna sinistra (Pfeil) 


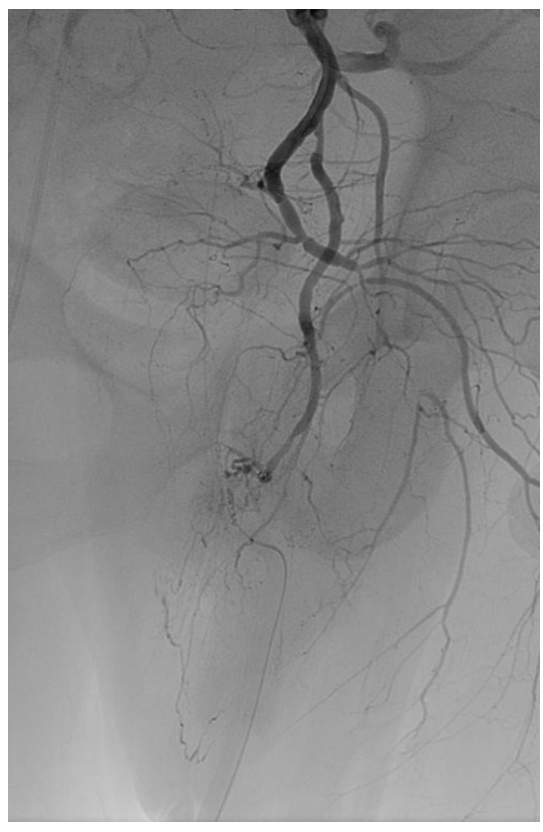

Abb. 3 A Kontrolle nach Coiling der A. pudenda interna sinistra

röhre, welche auch bereits Auswirkungen auf den Hämoglobinwert hatten.

\section{Diagnose}

Aufgrund der rezidivierenden unklaren Blutungen aus der Harnröhre wurde eine Zystoskopie durchgeführt. Hier zeigte sich eine pulsatile Masse im Bereich der bulbären Harnröhre. Zur genaueren Evaluierung wurde zusätzlich eine computertomographische Angiographie (CTA) des Beckens durchgeführt. Hier zeigte sich eine deutliche Kontrastmittelfahne in Richtung linker Peniswurzel, passend zu einer Gefäßverletzung oder einem Pseudoaneurysma (• Abb. 1). Der Patient wurde aufgrund dieses Befunds an die Abteilung für Urologie und Andrologie des Landeskrankenhauses Salzburg zur weiteren Therapie transferiert.

\section{Therapie und Verlauf}

Bei Eintreffen an unserer Abteilung kam es erneut zum vollständigen Sistieren der Blutung, weshalb eine radiologische Intervention vorerst nicht möglich war. Im Rahmen des weiteren stationären Aufenthalts kam es abermals zu einer massiven Blutung aus der Harnröhre. Initial wurde bei liegendem Dauerkatheter ein

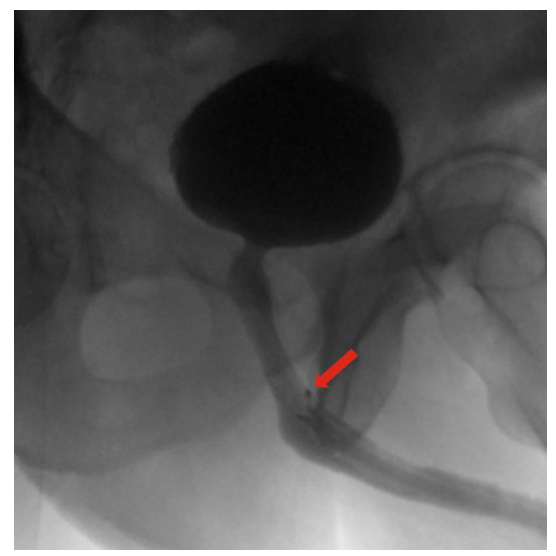

Abb. 4 A Miktionszystourethrogramm, postinterventionelle Kontrolle, Coils in der A. pudenda interna sinistra sichtbar (Pfeil)

Druckverband am Penis aufgebracht, wodurch die Blutung jedoch nicht gestoppt werden konnte und es wurde entschieden, eine Angiographie durchzuführen. In der Angiographie der linken Arteria (A.) iliaca interna stellte sich eine aktive Blutung aus einem Ast der A.pudenda interna (• Abb. 2), direkt angrenzend an den liegenden Harnblasenkatheter, dar. Die Blutung konnte mittels Embolisation durch Coils in derselben Sitzung versorgt werden (• Abb. 3). Nach dem Verschluss des entsprechenden Astes war in der anschließenden Kontrollangiographie keine aktive Blutung mehr nachweisbar. Ergänzend wurde auch eine Kontrollangiographie der rechten A. iliaca interna durchgeführt, hier ergab sich kein Hinweis für eine Blutungsquelle.

Der weitere postinterventionelle Verlauf war komplikationslos. Eine anschießende Miktionszystourethrographie zeigte einen unauffälligen Verlauf der Harnröhre und direkt angrenzend an die Harnröhre waren die eingeführten Coils sichtbar (• Abb. 4). Der Patient konnte nach Entfernung des Harnröhrenkatheters restharnfrei miktionieren und wurde nach Hause entlassen. Eine erneute Makrohämaturie trat nicht auf.

\section{Diskussion}

Oberflächliche Verletzungen der Harnröhre durch die Anlage eines Blasenkatheters sind eine häufige Komplikation und können meist konservativ behandelt werden. In unserem Fall kam es trotz Ver-
Urologe 2018 · 57:1357-1359

https://doi.org/10.1007/s00120-018-0665-8

(c) Der/die Autor(en) 2018

H. Griessner $\cdot$ T. Kunit $\cdot$ D. Colleselli .

M. Drerup - L. Oberhammer .

T. Wolfgruber $\cdot$ L. Lusuardi .

M. Mitterberger

\section{Pseudoaneurysma der bulbären Harnröhre nach traumatischer Katheterisierung}

\section{Zusammenfassung}

Wir präsentieren einen Fall eines 57jährigen Patienten mit intermittierenden $\mathrm{Hb}$ (Hämoglobin)-wirksamen Blutungen aus der Harnröhre nach traumatischer Katheteranlage. Ursächlich für die Blutung war ein arterielles Pseudoaneurysma in der bulbären Harnröhre. Die Blutungsquelle konnte mittels Angiographie dargestellt und embolisiert werden.

Schlüsselwörter

Pseudoaneurysma · Harnröhrenverletzung latrogen - Transurethraler Katheter . Intermittierende Blutung

\section{Urethral pseudoaneurysm after catheter-associated trauma}

\section{Abstract}

We present a case of heavy intermittent urethral bleeding in a 57-year-old man after traumatic catheterization caused by an urethral pseudoaneurysm. The source of bleeding could be detected by angiography and a superselective embolization was performed.

\section{Keywords}

Pseudoaneurysm · Urethral injury · latrogenic disease $\cdot$ Transurethral catheter $\cdot$ Intermittent bleeding

sorgung mit einem Dauerkatheter zu intermittierenden starken Blutungen. Initial ist die Durchführung einer Zystoskopie zur Lokalisation der Blutungsquelle eine gute diagnostische Methode. Bei akuter Blutung eignet sich auch eine Angio-CT zur weiteren Diagnostik. In unserem Fall bestätige sich der Verdacht eines Pseudoaneurysmas der A.pudenda interna sowohl im Angio-CT (• Abb. 1) als auch in der Zystoskopie. Da es bisher nur vereinzelt Berichte über Pseudoaneurysmen 
der Harnröhre gibt, ist die Frage der besten Behandlungsmethode aktuell noch nicht eindeutig geklärt. In einem Fallbericht von 2013 [6] wurde ein bulbäres Pseudoaneurysma transurethral koaguliert. Um die Gefahr einer späteren Harnröhrenstriktur zu reduzieren, war unserer Ansicht nach die superselektive Embolisation die geeignetere Therapie. In Medline fanden sich insgesamt drei publizierte Fälle [1, 3, 4], bei denen bisher eine Embolisation eines Astes der A.pudenda interna nach Bildung eines Pseudoaneurysmas erfolgte. In allen Fällen konnte dieser Eingriff komplikationslos durchgeführt werden und die Blutungsquelle erfolgreich ausgeschaltet werden. Insgesamt besteht eine sehr geringe Rate an Komplikationen bei der superselektiven Embolisation von Gefäßen. Bei der gezielten arteriellen Embolisation bei akuten gastrointestinalen Blutungen besteht eine Komplikationsrate von $<2 \%$. Ursächlich hierfür sind meistens kleine, häufig asymptomatische selbstlimitierende Ischämien. Die Kontraindikationen beschränken sich im Wesentlichen auf Kontraindikationen gegen jodhaltiges Kontrastmittel, welche eine entsprechende Vorbereitung des Patienten erfordern, sowie Kontraindikationen gegen eine arterielle Punktion. Für die Lokalisation und entsprechende Intervention ist eine aktive Blutung Voraussetzung [5].

Die traumatische Anlage bzw. lange Liegedauer eines transurethralen Blasenkatheters kann in seltenen Fällen zur Bildung eines Pseudoaneurysmas und in weiterer Folge zu starken Blutungen führen. Insbesondere bei intermittierenden Blutungen sollte an diese Diagnose gedacht werden. Therapeutisch ist unserer Ansicht nach in Anbetracht der möglichen Langzeitkomplikationen die superselektive Embolisation des betroffenen Gefäßes der transurethralen Koagulation überlegen.

\section{Fazit für die Praxis}

\footnotetext{
- Zur genaueren Differenzierung unklarer Blutungen aus der Harnröhre/ Harnblase ist die Zystoskopie eine einfache und effiziente Methode.
}

- Bei intermittierenden starken Harnröhrenblutungen nach Anlage eines Blasenkatheters sollte auch an ein Pseudoaneurysma in der Harnröhre gedacht werden.

- Zur Behandlung eines Pseudoaneurysmas in der Harnröhre ist die superselektive Embolisation des betroffenen Gefäßes unserer Meinung nach einer transurethralen Koagulation vorzuziehen.

\section{Korrespondenzadresse}

H.Griessner
Department of Urology
and Andrology, Paracelsus
Medical University Salzburg
Salzburg, Austria
h.griessner@salk.at

Funding. Open access funding provided by Paracelsus Medical University.

\section{Einhaltung ethischer Richtlinien}

Interessenkonflikt. H. Griessner, T. Kunit, D. Colleselli, M. Drerup, L. Oberhammer, T. Wolfgruber, L. Lusuardi und M. Mitterberger geben an, dass kein Interessenkonflikt besteht.

Dieser Beitrag beinhaltet keine von den Autoren durchgeführten Studien an Menschen oder Tieren. Alle Patienten, die über Bildmaterial oder anderweitige Angaben innerhalb des Manuskripts zu identifizieren sind, haben hierzu ihre schriftliche Einwilligung gegeben.

Open Access. Dieser Artikel wird unter der Creative Commons Namensnennung 4.0 International Lizenz (http://creativecommons.org/licenses/by/4.0/deed. de) veröffentlicht, welche die Nutzung, Vervielfältigung, Bearbeitung, Verbreitung und Wiedergabe in jeglichem Medium und Format erlaubt, sofern Sie den/die ursprünglichen Autor(en) und die Quelle ordnungsgemäß nennen, einen Linkzur Creative Commons Lizenz beifügen und angeben, ob Änderungen vorgenommen wurden.

\section{Literatur}

1. Bettez M, Aubé M, El Sherbiny M et al (2017) A bulbar artery pseudoaneurysm following traumatic urethral catheterization. Can Urol Assoc J. https://doi.org/10.5489/cuaj.4050

2. Karami H, Heidari F (2009) Pseudoaneurysm following percutaneous nephrolithotomy. Urol J 1:280-281

3. Radhakrishnan S, Marsh R, Sheikh N et al (2005) Urethral catheter induced pseudoaneurysm of the bulbar artery. Int J Urol. https://doi.org/10.1111/j. 1442-2042.2005.01176.x
4. Singh SK, Wadhwa P, Bapuraj JR et al (2005) Transcatheter embolization of internal pudendal artery pseudoaneurysm following traumatic urethral catheterization. Int Urol Nephrol. https:// doi.org/10.1007/s11255-004-4698-0

5. Walker TG, Salazar GM, Waltman AC (2012) Angiographic evaluation and management of acute gastrointestinal hemorrhage. World J Gastroenterol 18:1191-1201

6. Yuki H, Fuse M, Masuda A et al (2013) Urethral arterial bleeding from an iatrogenic pseudoaneurysm due to catheterization. Med Surg Urol 2:2 